\title{
Analyse exploratoire de l'intégration des jeunes migrants qualifiés dans les métropoles de Montréal (Québec) et Porto Alegre (Rio Grande do Sul, Brésil) \\ Exploratory analysis of the integration of educated young adult migrants in the cities of Montréal (Québec) and Porto Alegre (Rio Grande do Sul, Brazil) \\ Análisis exploratorio de la integración de jóvenes migrantes calificados en las ciudades de Montréal (Québec) y Porto Alegre (Rio Grande do Sul, Brasil)
}

\section{Grazielle Betina Brandt et Serge Côté}

\section{Volume 57, numéro 161, septembre 2013}

Version originale soumise en avril 2013. Version révisée reçue en janvier 2014.

URI : https://id.erudit.org/iderudit/1024900ar

DOI : https://doi.org/10.7202/1024900ar

Aller au sommaire du numéro

Éditeur(s)

Département de géographie de l’Université Laval

ISSN

0007-9766 (imprimé)

1708-8968 (numérique)

Découvrir la revue

Citer cet article

Brandt, G. B. \& Côté, S. (2013). Analyse exploratoire de l'intégration des jeunes migrants qualifiés dans les métropoles de Montréal (Québec) et Porto Alegre (Rio Grande do Sul, Brésil). Cahiers de géographie du Québec, 57(161), 193-208. https://doi.org/10.7202/1024900ar

\section{Résumé de l'article}

Dans le cadre d'une recherche exploratoire sur la migration interne des jeunes qualifiés, nous nous sommes intéressés au processus de leur intégration en milieu métropolitain et avons tenté d'identifier les modes d'appropriation réelle et symbolique de l'espace que les jeunes pratiquent tout au long de leur trajectoire. Pour certains jeunes qui migrent, l'installation en milieu métropolitain suscite quelques appréhensions. Pour d'autres, leur insertion dans la métropole se présente comme une expérience enrichissante et valorisée des points de vue social et culturel. Nos observations sur le parcours d'intégration dans l'espace métropolitain proviennent d'un corpus d'entrevues avec des jeunes qualifiés du Brésil (20 entrevues) et du Québec (20 entrevues). Nous avons cherché à documenter les difficultés éprouvées par les jeunes, les stratégies qu'ils ont mises en oeuvre et les soutiens dont ils ont pu bénéficier pour réaliser leur processus d'intégration. 


\section{Analyse exploratoire de l'intégration des jeunes migrants qualifiés dans les métropoles de Montréal (Québec) et Porto Alegre (Rio Grande do Sul, Brésil)}

\author{
Exploratory analysis of the integration of \\ educated young adult migrants in the cities \\ of Montréal (Québec) and Porto Alegre \\ (Rio Grande do Sul, Brazil)
}

Análisis exploratorio de la integración de jóvenes migrantes calificados en las ciudades de Montréal (Québec) y Porto Alegre (Rio Grande do Sul, Brasil)

\author{
Grazielle BETINA BRANDT \\ Programme en développement régional de \\ I'Université de Santa Cruz do Sul (UNISC), Brésil \\ Grazielle@unisc.br
Serge CôTÉ
Centre de recherche sur le développement territorial, Université du Québec à Rimouski (UQAR) \\ Serge_Cote@uqar.ca
}

\section{Résumé}

Dans le cadre d'une recherche exploratoire sur la migration interne des jeunes qualifiés, nous nous sommes intéressés au processus de leur intégration en milieu métropolitain et avons tenté d'identifier les modes d'appropriation réelle et symbolique de l'espace que les jeunes pratiquent tout au long de leur trajectoire. Pour certains jeunes qui migrent, l'installation en milieu métropolitain suscite quelques appréhensions. Pour d'autres, leur insertion dans la métropole se présente comme une expérience enrichissante et valorisée des points de vue social et culturel. Nos observations sur le parcours d'intégration dans l'espace métropolitain proviennent d'un corpus d'entrevues avec des jeunes qualifiés du Brésil (20 entrevues) et du Québec (20 entrevues). Nous avons cherché à documenter les difficultés éprouvées par les jeunes, les stratégies qu'ils ont mises en œuvre et les soutiens dont ils ont pu bénéficier pour réaliser leur processus d’intégration.

\section{Mots-clés}

Migration interne, jeunes qualifiés, intégration, milieu métropolitain.

\section{Abstract}

In the context of an exploratory study on internal migration among educated young people, our research examines the processes involved in their integration within unfamiliar metropolitan areas and analyzes them to identify patterns in the real and symbolic strategies they adopt to integrate successfully into the environments they have moved to. For some young migrants, moving to a metropolis creates feelings of apprehension. Others view their integration into the city as an enriching experience and an opportunity for social and cultural development. Our observations about the process of integration into metropolitan areas are drawn from a corpus 
of interviews with educated young adult migrants from Brazil (20 interviews) and from Québec (20 interviews). The study documents the challenges faced by the interviewees, the strategies they adopted and the support they received in the course of their integration process.

\section{Keywords}

Internal migration, young educated migrants, integration, metropolitan area.

\section{Resumen}

Durante una investigación exploratoria sobre la migración interna de jóvenes calificados, nos hemos interesado en el proceso de su integración en medio metropolitano y hemos tratado de conocer los modos de apropiación real y simbólica del espacio practicados por los jóvenes durante su trayectoria. Ciertos jóvenes migrantes aprehenden instalarse en medio metropolitano. Para otros, su inserción en la metrópoli se presenta, desde el punto de vista social y cultural, como una experiencia rica y valorizadora. Nuestras observaciones de la trayectoria de integración en medio metropolitano provienen de un corpus de entrevistas con jóvenes calificados de Brasil (20 entrevistas) y de Québec (20 entrevistas). Hemos tratado de documentar las dificultades que enfrentaron los jóvenes, sus estrategias et el soporte que ellos recibieron para concretizar el proceso de integración.

\section{Palabras claves}

Migración interna, jóvenes calificados, integración, medio metropolitano.

\section{Introduction}

Les grands centres urbains attirent encore une partie appréciable des jeunes, surtout ceux qui cherchent à se qualifier ou qui possèdent un bon niveau de qualification. La ville - et surtout la grande ville - rend possibles une promotion sociale et un avancement professionnel plus intéressants, et une qualité de vie en accord avec des aspirations liées à la consommation (Bujold et Gauthier, 1995 : 37). Comme le montrent Fréchette et al. (2004: 85-86), la migration vers les grandes villes canadiennes initie les jeunes à l'expérience de la ville, «expérience de confrontation à la nouveauté puis, dans la plupart des cas, de familiarisation progressive». Pour le cas du Brésil, Oliveira et Da Mata (2008: 03) ont tenté d’identifier les caractéristiques déterminantes qui amènent les jeunes qualifiés à migrer vers certaines villes brésiliennes. Leur recherche révèle que les villes qui veillent au bien-être et à la qualité de vie de leurs résidants exercent une forte attraction sur les migrants qualifiés, comme c'est le cas pour les villes à faible degré d'inégalité sociale et à faible indice de violence. Oliveira et Da Mata (2008: 10) ont observé que le revenu attendu est une variable importante dans la prise de décision du travailleur et dans la détermination de son niveau de bienêtre, mais n'est pas la seule raison. Des facteurs qui vont au-delà de la dynamique du marché du travail exercent une influence considérable quand un travailleur instruit décide de se fixer. La recherche a permis de constater qu'il existe une relation directe entre le niveau d'éducation de la population résidante dans la ville et l'attraction que celle-ci exerce sur des migrants qualifiés: les qualifiés tendent à aller vers les localités où l'on trouve un plus haut niveau de scolarité. Cette tendance confirme l'hypothèse de l'existence «d'externalités» positives associées au capital humain. 
Au cours des dernières décennies, le Brésil a connu de forts mouvements migratoires vers les grandes métropoles du pays. Actuellement, les mouvements de la population se font selon d'autres patrons. Matos et Braga (2005: 25) identifient de nouveaux mouvements populationnels surtout vers les régions littorales des États brésiliens et vers les villes moyennes. Au Québec, comme le montrent les rapports du Groupe de recherche sur la migration des jeunes (Gauthier et al., 2001 et 2006), les grandes motivations de déplacement des jeunes Québécois sont d'aller étudier, de vivre sa vie dans un nouveau milieu ou encore de retourner parmi leurs proches dans leur région d'origine. Dans le cas des jeunes Québécois, Fréchette et al. (2004 : 100) démontrent que la migration "n'est pas ni [sic] unidirectionnelle, ni associée à la sédentarité». Une partie de cette jeunesse en mouvement préfère simplement «séjourner» dans le milieu métropolitain plutôt que de s'y installer définitivement. Nous remarquons, à partir de recherches récentes, comme celles de Potvin (2006) et LeBlanc (2010), que le phénomène de la migration de retour est déjà une réalité pour les jeunes Québécois. Les préoccupations concernant le départ des jeunes sont encore inscrites à l'ordre du jour des chercheurs québécois, néanmoins, avec les perspectives ouvertes par la migration de retour, les recherches débouchent sur des thématiques plus complexes qu'auparavant.

Pour cerner les diverses facettes de l'intégration des jeunes migrants qualifiés en milieu métropolitain, nous examinerons, à partir d'une analyse exploratoire, les stratégies qu'ils utilisent. Cela nous permettra de saisir leurs interactions urbaines et le sens accordé à ces interactions lors de la mobilisation de ressources variées (économiques, sociales, culturelles). Chaque jeune a accès à des ressources différenciées, autant matérielles que symboliques. Il devient important, dans notre étude, de souligner l'arrière-plan où ces jeunes mènent leurs activités, ce qui nous permet de mieux cerner la complexité et la diversité des environnements sociaux où ils évoluent.

\section{Revue de littérature}

La plupart des travaux sur les migrations vers les métropoles concernent la migration internationale. Même si les modèles proposés pour décrire l'intégration des immigrants provenant de l'étranger ne peuvent convenir tels quels pour rendre compte de l'intégration des personnes dont le déplacement est une migration interne, certains aspects des deux types de migration se rejoignent. Plusieurs constats de chercheurs s'appliquent autant à un type de migration qu'à l'autre. Ainsi en est-il des affirmations à l'effet que «les grandes villes sont le creuset de l'intégration» (Papademetriou, 1997: 140); que l’intégration propre aux époques dominées par l'économie manufacturière assurait aux nouveaux arrivants dans les métropoles des perspectives de promotion sociale qui ne sont plus aussi abondantes maintenant que l'«économie [est] dominée par les industries de services à forte concentration de savoir qui exigent habituellement un niveau de scolarité postsecondaire» (Waldinger, 1997: 37), donc une qualification préalable plus substantielle; que les grandes agglomérations sont un des terrains où la mondialisation se joue le plus intensément, «ces centres urbains [étant] devenus le théâtre stratégique de toute une série de conflits et de contradictions» (Sassen, 1997: 35). Dans ces conditions, les dirigeants métropolitains font face à des défis considérables de «gouvernance» quand vient le temps d'assurer une cohésion sociale minimale dans les grands ensembles urbains (OCDE, 2001 : 33). 
Quand Chambers (1994: 23) avance que «the modern metropolitan figure is the migrant», il souligne le fait que toutes les métropoles ont en commun d'avoir une composition démographique hétérogène sur les plans des origines géographiques, des traditions culturelles ou professionnelles, des langues et des accents, cette diversité étant nourrie par le brassage incessant des populations, par la constitution de marges et de niches au sein des territoires urbains, ainsi que par la fluidité même des rapports sociaux dans les grands ensembles humains que constituent les métropoles.

Il est inévitable que les arrivants doivent s'adapter à un nouvel environnement lorsqu'ils s'installent dans une métropole, surtout s'ils sont originaires de collectivités plutôt homogènes sur le plan culturel. Chez les immigrants provenant de l'étranger, le processus est souvent long et complexe et ne se complète parfois qu'à la deuxième génération. Chez les migrants internes, l'intégration présente quelques points de similitude avec celle des immigrants, en ce sens que la plupart des individus doivent s'adapter à des us et coutumes nouveaux et doivent également «négocier» certains éléments de leur identité pour s'en forger une qui convient mieux au nouveau contexte métropolitain (Breton, 1994: 247), ce qui n'exclut pas de garder des liens avec son milieu d'origine et de continuer d'être influencé par lui.

Les propos les plus fréquemment tenus pour expliquer les migrations internes veulent que les flux migratoires soient modulés par les besoins du marché du travail. Or, ce qui peut sembler à certains l'évidence même fait souvent l'objet de mises au point qui relativisent la puissance de cette cause supposée. Au terme d'une analyse approfondie de l'insertion des jeunes diplômés en France, menée avec les outils de l'analyse géographique, Perret (2008: 83) avance qu'«expliquer les situations migratoires régionales par des déséquilibres entre des économies locales, suivant ainsi les théories de l'attraction et de la répulsion, [...] apparaît difficile» en raison des nombreux cas qui ne concordent pas avec le modèle. Certains économistes ont remis en cause l'aspect dominant de ce modèle en soutenant que les contraintes du marché de l'emploi ne font pas foi de tout et qu'il faut se persuader que «there is evidence that "soft factors" such as attitudes, perceptions and indentities affect economic behaviour more than they are driven by them» (Constant et Zimmermann, 2011 : 163). Cette posture théorique fait en sorte que les facteurs responsables de l'intégration des migrants qui s'installent en milieu métropolitain concernent bien plus que les modalités de l'insertion professionnelle. Outre l'accès au marché du travail, plusieurs autres facteurs interviennent dans le processus d'intégration des migrants. C'est ce que constataient, en tout cas, il y a plus de 40 ans, les chercheuses Messier et Marois (1972) dans leur étude sur la migration des Gaspésiens dans la métropole montréalaise: les liens de sociabilité, l'accès aux services, les conditions de logement jouaient aussi un rôle crucial dans l'adaptation des migrants à leur nouvelle situation dans la métropole.

Les zones métropolitaines ont longtemps constitué une destination attrayante pour les jeunes migrants qualifiés qui souhaitent y réaliser leurs projets de vie, et elles le demeurent encore largement aujourd'hui. Ainsi, les zones métropolitaines au Québec et certaines d'entre elles au Brésil agissent comme agents socialisateurs pour cette jeunesse en mouvement.

Selon Desmarais et al. (2001: 109), «l'expérience de l'intégration comprend trois étapes: 1) l'arrivée; 2) la familiarisation, les premières impressions et les premières expériences; 3) la vie actuelle et les projets». À la suite de ces chercheurs, nous 
considérons l'intégration comme le processus par lequel les individus mobilisent les ressources nécessaires à leur installation et à leur maintien dans un milieu donné. Nous distinguons la dimension professionnelle, la dimension sociale et la dimension symbolique. La première touche l'acquisition des compétences et l'insertion dans le marché du travail; la deuxième concerne l'insertion sociale des individus qui se concrétise, par exemple, dans l'accès aux services, l'affiliation aux réseaux existants, la capacité de susciter des interactions et de créer des relations avec les habitants de la ville, etc.; la troisième considère les représentations des acteurs, les coutumes des habitants et les valeurs attachées aux lieux et aux façons de faire propres à une ville donnée. L'intégration repose sur des éléments matériels concrets et suppose en particulier l'établissement de rapports avec l'espace urbain (Desmarais et al., 2001). Mais elle comprend aussi des aspects subjectifs et fluctue selon le vécu des individus.

\section{Démarche de recherche et caractéristiques de l'échantillon des répondants}

Notre recherche a visé en particulier les jeunes âgés de 20 à 34 ans qui sont soit étudiants en train de réaliser un parcours universitaire, soit jeunes diplômés de l'enseignement supérieur ayant accédé au marché du travail. Nous avons utilisé le modèle boule de neige (Goodman, 1961) pour la procédure de sélection des jeunes. Quarante entrevues qualitatives ont été réalisées au total (20 dans la métropole de Porto Alegre et 20 dans la métropole de Montréal), constituant l'échantillon de notre recherche.

Un contact par téléphone ou par courrier électronique a été établi avec les participants à la recherche. Chacun des participants a eu la liberté de choisir le lieu de l'entrevue (à la maison, dans un espace public, dans une université, etc.). Les entrevues ont été réalisées individuellement et la participation a été volontaire. Les participants étaient libres de se retirer sans préjudice et en tout temps, sans devoir justifier leur décision.

Les entrevues ont été menées de janvier à juillet 2009. Elles ont été réalisées en français pour les jeunes Québécois et en portugais pour les jeunes Brésiliens. Les entrevues réalisées en portugais ont été traduites en français avant l'analyse. Leur durée moyenne a été d'une heure à une heure et demie. Dans la narration de l'expérience migratoire, une attention particulière a été accordée aux étapes de la migration (période avant le déplacement; moment du déplacement et de l'installation; moment actuel et projections d'avenir), étant donné l’importance prévisible de ces étapes dans l'explication du processus d'intégration.

L'échantillon total comprend 23 hommes et 17 femmes, près de la moitié (48\%) âgés de 30 à 34 ans. Les 25-29 ans représentent 42\% de l'échantillon et les 20-24 ans en forment 10\%. La plupart (70\%) se disaient célibataires au moment de l'entrevue, alors que 20\% avaient un conjoint de fait et 10\% étaient mariés. La majorité (95\%) n’avait pas d’enfant. Les interviewés exerçant une activité rémunérée représentaient 83\% de l'échantillon.

Tous ces jeunes avaient en commun d'être originaires d'une localité située dans une région non métropolitaine du Rio Grande do Sul ou de la province de Québec et d'avoir un parcours migratoire les ayant conduits, entre autres lieux, dans la métropole de 
Porto Alegre ou dans celle de Montréal. Méthodologiquement, nous avons considéré qu'il y a migration quand un jeune s'installe dans une localité autre que celle dans laquelle se trouvaient ses parents quand il cohabitait avec eux.

\section{Profil sociodémographique de Montréal et de Porto Alegre}

Notre travail porte sur la migration interrégionale des jeunes qualifiés dans les métropoles de Montréal et Porto Alegre comme lieux de destination. Les deux villes sont d'importants pôles universitaires qui attirent des étudiants. Dans les études récentes, tant au Brésil qu'au Canada (Fréchette et al., 2004; Molgat et Saint-Laurent, 2004; Abramo et Branco, 2005 ; Correa, 2008), on a observé également dans les deux villes la difficulté des jeunes à se définir une identité urbaine.

Capitale de l'État le plus méridional du pays, le Rio Grande do Sul, la métropole de Porto Alegre constitue la plus grande région métropolitaine du sud du pays et la quatrième plus peuplée du Brésil. La ville de Porto Alegre compte 1467823 habitants et la région métropolitaine, 4011224 (IBGE, 2011). Sur le plan démographique, Porto Alegre a connu à partir de la décennie 1970, selon l'étude d'Alonso et Brinco (2006), un intense flux migratoire, causé en grande partie par les changements structurels qui se sont produits dans le secteur agricole et qui ont engendré une migration vers les grands centres urbains. Les deux auteurs soulignent que la ville, tout en étant la capitale de l'État et un centre dynamique au plan économique, est celle qui reçoit le plus de migrants en provenance d'autres villes de l'État et le plus de personnes qui effectuent une migration intramétropolitaine.

Dans une étude sur la mobilité urbaine métropolitaine, Peixoto et Mello (2009) évaluent que les groupes d'âge qui se situent dans la fourchette de 20 à 60 ans affichent une mobilité supérieure à la moyenne de l'État du Rio Grande do Sul. Selon la Recherche mensuelle d'emploi de l'IBGE (avril 2009), la proportion que représente la population active dans l'ensemble de la population de 15 ans et plus (taux d'activité) était de 55,8\% au moment de la recherche, soit $65 \%$ pour les hommes et $47,9 \%$ pour les femmes.

Selon le Profil sociodémographique de Montréal (2008), depuis les années 1970, le développement démographique de cette ville s'est effectué en trois phases: une période de déclin, entre 1971 et 1986; une période de stabilité, entre 1986 et 1996; et une période de croissance, de 1996 à 2006. L’agglomération de Montréal comptait 1981672 habitants au recensement de 2011 et sa région métropolitaine, 3824221. Selon l'Institut de la statistique du Québec (2013), la population de Montréal se distribue de la façon suivante selon les tranches d'âge:

$\begin{array}{ll}\text { 0-14 ans } & 298063 \text { habitants } \\ \text { 15-24 ans } & 232468 \text { habitants } \\ \text { 25-44 ans } & 643787 \text { habitants } \\ \text { 45-64 ans } & 506499 \text { habitants } \\ 65 \text { ans et plus } & 300855 \text { habitants }\end{array}$

Le solde migratoire interrégional pour la période 2011-2012 est de - 20492 habitants. 


\title{
Analyse exploratoire des stratégies en œuvre dans le processus d'intégration des jeunes migrants qualifiés en milieu métropolitain
}

L’intégration des migrants dans les localités d'accueil est un processus complexe et à facettes multiples. Au Québec, un certain nombre de travaux ont été effectués sur le sujet des jeunes migrants internes et de leur intégration dans la grande ville (Boudreault et Parazelli, 2004; Fréchette et al., 2004; Molgat et Saint-Laurent, 2004). Les métropoles sont sans doute un lieu de passage pour une partie des jeunes. En fait, plusieurs facteurs entrent en jeu dans le déroulement du parcours migratoire des jeunes: leurs motivations à s'installer dans un grand centre, l'envie de migrer à nouveau après avoir réalisé un premier déplacement, les rapports établis avec le milieu métropolitain, etc. Idéalement, tous ces éléments devraient être pris en compte ensemble pour comprendre adéquatement le mouvement migratoire des jeunes.

L'intégration des jeunes migrants dans les grands centres se construit surtout à partir des éléments structurels et institutionnels qui se présentent à eux. Par ailleurs, les trajectoires personnelles et les réseaux de relations constituent des terrains riches pour comprendre la question de l'intégration des migrants. Il y a donc nécessité de se tourner vers des perspectives multiples afin de bien cerner la question de l'intégration des migrants. Cette partie de notre travail de recherche va permettre de connaître de façon plus précise les conditions d'intégration des jeunes migrants à partir de leurs stratégies économiques et sociales.

\section{L'intégration des jeunes Brésiliens qualifiés à Porto Alegre}

D’après les témoignages des jeunes migrants brésiliens, l'expérience de migrer vers la métropole est appréciée de façon fort diverse selon les individus. Le moment de l'installation à Porto Alegre et le temps écoulé depuis ont laissé, selon les témoignages recueillis, autant de bons que de mauvais souvenirs. Dans notre recherche, il est important d'observer que l'intégration sociale des jeunes migrants qualifiés fait référence à la façon dont les jeunes ont vécu leurs trajectoires migratoires individuelles, du point de vue de leurs différentes conditions migratoires. Un premier migrant reconnaît les contraintes économiques qui étaient les siennes à l'époque de son adaptation et de son insertion à Porto Alegre.

\begin{abstract}
Du point de vue strictement matériel, nous avons déménagé parce que mon épouse et moi, nous avons obtenu une bourse pour faire la maîtrise à Porto Alegre. Sans cette bourse, nous n'aurions pas migré. Nos familles n'ont pas d'immeubles à Porto Alegre. Nos familles n'avaient pas les moyens de nous aider financièrement à l'époque. J'avoue que j'ai beaucoup souffert pour m'adapter à cette situation matérielle limitée. L'environnement n'était pas des plus agréables, alors la situation a été difficile... pas traumatique, mais elle a été difficile $(\mathrm{MI}-04 / \mathrm{H})^{1}$.
\end{abstract}

La situation de ce jeune Brésilien n’est pas unique. Les problèmes d'ordre économique touchent plusieurs jeunes qualifiés au Brésil où les difficultés à joindre les deux bouts ne sont pas l'exclusivité des jeunes migrants peu scolarisés. La vulnérabilité économique conditionne de façon décisive le délai d’insertion des migrants qualifiés.

1 De manière à respecter l'anonymat des jeunes interviewés, nous avons opté pour une codification composée par le sigle MI suivi d'un numéro d'ordre servant à identifier le jeune migrant dans la banque de données (MI-01, MI-02, MI-03... MI-40) ainsi que du sexe du jeune (F ou H). 
Des études empiriques au Brésil (Pereira, 2000: 55) montrent que, dans ce pays, ce ne sont ni les personnes les plus pauvres et les moins scolarisées, ni les plus riches et les plus scolarisées qui migrent, mais plutôt celles avec un statut intermédiaire, tant en termes scolaires que monétaires. Ce groupe réalise les plus grands échanges sociaux et économiques dans le mouvement migratoire.

Une fois franchie la période critique de l'installation - plus ou moins longue selon les individus -, l'insertion socioprofessionnelle du migrant qualifié assure son intégration à long terme dans la métropole, comme ce fut le cas pour ce même jeune:

\begin{abstract}
Cela en a valu la peine. Je le ferais de nouveau, malgré les difficultés, malgré les regrets. Je trouve vraiment que cela a valu la peine, j'ai eu des occasions de travail que je n'aurais peut-être pas eues à (municipalité d'origine). Des occasions d'apprentissage et de formation que je n'aurais pas eues si j'étais resté à (municipalité d'origine). Bons moments de réussite que je n’aurais pas eus à (municipalité d'origine). (MI-04/H)
\end{abstract}

Les jeunes migrants qualifiés rencontrés au Brésil ne forment pas un ensemble homogène dans notre échantillon, car ils sont marqués par des différences et des inégalités dues surtout au niveau de revenu de leur famille d’origine. Pour certains, le soutien financier parental représente la source principale de revenu, et ce, même s'ils exercent une activité salariée. En comparaison, chez les jeunes migrants québécois, c'est le travail qui constitue la première source de revenu. Au Brésil, le salaire de stagiaire, ou même une bourse de recherche, ne suffisent pas à couvrir l'ensemble des dépenses dans une métropole comme Porto Alegre.

Dans les familles les plus fortunées, il existe une sorte de solidarité familiale qui facilite grandement le processus d'insertion sociale des migrants à Porto Alegre sous la forme, notamment, de l'aide au logement:

\footnotetext{
Pour moi, ça a été facile. Peut-être justement, du fait d'avoir un lieu pour vivre, d'avoir un appartement bien localisé et d'avoir ma sœur ici à Porto Alegre et de savoir que je pourrais compter sur elle. (MI-08/F)

Je trouve que ça n'a pas été difficile. Quand j’ai quitté (municipalité d'origine), mon frère, qui habite là-bas maintenant, à l'époque habitait à Porto Alegre. Alors je n'ai pas eu de grands problèmes d'adaptation. (MI-02/H)

L'installation et l'adaptation ont été relativement faciles. J'avais mes oncles et mes cousins qui habitaient ici... Ça a été facile pour moi, j’ai vécu avec eux au début. J’ai vécu deux à trois mois avec mes cousines et, ensuite, j'ai vécu un an et demi avec mes oncles. (MI-14/H)
}

Certains jeunes rencontrés peuvent donc compter sur l'appui et les ressources économiques disponibles au sein de leur famille afin de réaliser leur projet de s’installer à Porto Alegre. Une telle situation a été observée avec plus d’intensité chez les jeunes qui ont quitté pour la première fois le foyer parental pour étudier dans la métropole. Si la solidarité familiale joue un rôle majeur dans le processus d'intégration des jeunes migrants, d'autres éléments peuvent aussi y contribuer. Un de ces éléments est l'expérience de départ à l'étranger. À la suite de Garneau (2006: 23), nous constatons que l'appropriation ou la fréquentation préalable de l'espace international réduit les difficultés de l'intégration, notamment au moment de s'installer dans une métropole comme Porto Alegre. 
Je pense que comme j'avais vécu à l'étranger, connu des moments difficiles, j'ai l'impression que je me suis adapté très facilement à Porto Alegre. Je n’ai pas eu de grands problèmes. J'aime cette ville jusqu'à aujourd'hui. Je n’ai eu aucun problème avec elle, mais au début je profitais encore plus de Porto Alegre. (MI-06/H)

Les liens avec les amis jouent également un rôle dans l'acclimatation aux nouvelles réalités métropolitaines. Le réseau des relations dans le lieu de destination se constitue comme une ressource essentielle pour les migrants. À partir d'un réseau d'amis, le jeune arrivant peut accéder plus facilement à l'information, s'enquérir de diverses modalités d'action et surtout surmonter les difficultés du début:

Ça a été facile, très facile, parce que la plupart de mes amis d'enfance étaient déjà installés ici, alors je suis parti habiter près d'eux. L'adaptation n’a pas été trop difficile, surtout parce que j'avais des amis autour, j'avais une vie sociale établie. Je n'ai pas eu besoin de me faire des nouveaux amis. L'amitié s'est construite au fil des années autour de mes amis de longue date. (MI-01/H)

Parmi tous les liens interpersonnels, ce sont les relations amicales et parentales qui contribuent de la façon la plus déterminante à l'intégration sociale des jeunes migrants qualifiés brésiliens dans la métropole. Par ailleurs, la plupart des jeunes ont des liens avec des individus originaires de la même municipalité ou région d'origine qu'eux. Ce n'est pas toujours évident pour les jeunes migrants qualifiés de développer rapidement des liens interpersonnels avec d'autres jeunes de la métropole d'accueil :

Tu arrives à Porto Alegre, un grand centre, tout le monde a déjà sa vie propre. Tout le monde a déjà ses amis et personne ne s'ouvre aux gens. Alors, j'ai fini par m’approcher des gens que je connaissais déjà de (municipalité d’origine). Ça a pris du temps pour me faire quelques amis porto-alegrenses. (MI-12/F)

J'ai plusieurs amis qui viennent de (municipalité d'origine) et qui sont à Porto Alegre. J'ai beaucoup de contacts avec eux, surtout que j'ai des voisins de (municipalité d'origine). C'est un cercle d'amis, des gens qui viennent de ma région vers la capitale. (MI-20/F)

Malgré cette conscience aiguë des difficultés de l'intégration, certains répondants n'excluent pas la possibilité d'aller s'installer ailleurs et, donc, de vivre à nouveau les mêmes difficultés. Environ le quart des répondants brésiliens ont l'intention de quitter un jour Porto Alegre, tandis qu'un peu plus de la moitié affirment y penser parfois. En définitive, il n'y a qu'un cinquième de ces migrants qui ne pensent pas du tout à partir. Selon les témoignages de nos interviewés, la métropole de Porto Alegre ne se présente pas forcément comme un lieu où les jeunes s'établissent et s'enracinent, mais davantage comme un lieu de passage, comme une étape dans leur vie. Pour la plupart des jeunes, le choix de vivre dans une métropole ne semble pas être définitif.

L'individualisation des jeunes est un processus qui prend de plus en plus de place dans les métropoles mondiales. Correa (2008: 12) démontre que, pour les jeunes Brésiliens, le processus d'individualisation est inexorable et s'inscrit dans d'autres processus (massification culturelle, mondialisation et transformation de la société de consommation). En conséquence, cette plus grande liberté individuelle dans les grands centres urbains conduit à l'indifférence des habitants les uns à l'égard des autres (Simmel cité par Molgat et Saint-Laurent, 2004: 34). Nous constatons que l'indifférence et l'anonymat typiques des grandes villes peuvent avoir des effets négatifs dans le processus d’insertion sociale de certains jeunes qualifiés: 
Ça a été relativement difficile parce que je me sentais très seule à Porto Alegre. Je me suis rendu compte que les personnes étaient individualistes, égoïstes et ne pensaient qu'à eux-mêmes. La politique du bon voisinage ne marche pas très bien. Alors, dans ce sens, je me sentais un peu abandonnée... il y avait la question de la distance, mais ce qui me dérangeait le plus c'était ce manque de réceptivité. (MI-11/F)

\begin{abstract}
Ça a été très difficile! En premier, parce que ma famille ne voulait pas. En second, parce que les gens à Porto Alegre sont très fermés. Les gens à Porto Alegre n’ont pas la même affection que les gens de ma région. Il n'existe pas de contact ou d'approche, car le rythme de vie est plus intense, les gens travaillent, sont toujours à courir et ils n'ont pas de temps à consacrer aux autres. J'ai appris toute seule... J'ai cherché un travail, un appartement, et disons que je me sentais très seule. (MI-15/F)
\end{abstract}

Dans certains entretiens, la perception des liens sociaux faibles à Porto Alegre produit, sur certains jeunes qualifiés, un sentiment de rejet, voire de peur, qui tend à faire obstacle à leur processus d'insertion sociale. Ce même sentiment est vécu par les jeunes Québécois, surtout ceux qui partent du milieu rural et se dirigent vers les grandes villes, comme Montréal et Québec (Molgat et Saint-Laurent, 2004: 52). Ce sont les jeunes qui manifestent le plus fort attachement à leur région d'origine qui ont le plus tendance à souligner les aspects négatifs de la vie dans la métropole.

Concernant le processus d'intégration des jeunes migrants qualifiés au Brésil, nous constatons que différents défis se présentent à eux. Parfois c'est le manque de lieux de rencontre, parfois c'est la violence de la grande ville qui amène les jeunes à l'isolement. Ce peut être aussi des difficultés à accéder à certains services de la ville ou encore des difficultés financières. Cette jeune femme mentionne plusieurs éléments qui ont retardé son intégration:

Mon intégration à Porto Alegre a été très compliquée, par le fait d'être une grande ville, avec ses différentes habitudes. Tu as besoin d'apprendre à utiliser le transport public, à (municipalité d'origine) ce n'est pas nécessaire. Tu as besoin d'observer les horaires d'autobus. Tu ne peux pas être toute seule dans les arrêts d'autobus le soir. Ça dépend de l'endroit, mais tu ne peux plus y aller à certaines heures. Tu a toujours besoin de faire attention à cause de la violence; les personnes sont plus fermées en fonction de cela. Mes voisins, je ne les connais pas. Quelques-uns te saluent dans l'ascenseur, mais les gens d'ici sont complètement différents. (MI-12/F)

Porto Alegre est vue comme un milieu qui offre de nombreuses possibilités dans le parcours universitaire et socioprofessionnel de ces jeunes. Cependant, la violence et les problèmes liés à l'infrastructure se retrouvent chez tous dans leur représentation de la ville. Nous observons cependant que le soutien familial peut réduire la représentation négative des jeunes par rapport à la métropole. Cela diminue les difficultés économiques et affectives vécues lors de leur arrivée dans la métropole.

Toutefois, pour certains jeunes, l'intégration à Porto Alegre a été vécue de façon progressive, la transition vers la métropole s'étant faite par étapes. Ces deux extraits nous montrent le processus de transition:

Ça a été un processus - comment dirais-je? - progressif. Dans les premières années, j’étais très attaché à ma ville d'origine et à ma famille, alors j'avais besoin de retourner chez moi toutes les fins de semaine pour me ressourcer. Porto Alegre est une ville très différente de (municipalité d'origine) et, au départ, le rythme de vie m’épuisait. Il fallait que je prenne l'air... Donc j'allais à (municipalité d'origine). Mais, avec le temps, je me suis fait des amis ici et ma ville ne me manquait pas plus que ça. (MI-17/H) 
Ça a pris un certain temps avant que je m'adapte. J'étais loin de chez moi, mais aujourd'hui je trouve que je me suis adapté rapidement. On fait même des blagues à ce sujet, mes amis et moi, parce qu'on allait toute la fin de semaine à (municipalité d'origine). Mais, après une année, tu y vas au moins une fois par deux semaines. L’année suivante, une fois par mois et aujourd'hui, ça prend six mois pour y aller. (MI-12/F)

\section{L'intégration des jeunes Québécois qualifiés à Montréal}

L’intégration des jeunes Québécois à la métropole de Montréal est également observée sous divers aspects. La qualité du logement et le choix du quartier constituent des éléments qui influencent l'intégration des jeunes Québécois dans la ville. À partir des opinions que les jeunes formulent sur leur processus d'intégration, nous observons que cette situation peut aussi bien être positive que négative:

On avait un bel appartement sur le Plateau-Mont-Royal, je suis resté là pendant trois ans. Pour moi c'était une découverte de pouvoir tout faire à côté, tu es proche de la rue MontRoyal, j'ai adoré ça. Seule chose, c'était un peu loin de l’Université de Montréal. Mais j'ai adoré la ville. [...] Je pense que le fait qu'on sorte la fin de semaine, qu'on rencontre des gens m’a aidé encore plus à apprécier la ville. (MI-25/H)

Ici à Montréal, c'était un peu n'importe quoi [...].Donc je me suis ramassée à vivre avec quelqu'un que je ne connaissais pas. Et à Montréal, le grand défi c'est les appartements. C'est difficile de se trouver un appartement. Le mien c'était incroyable. Ce n'est pas si pire, mais c'était trop bruyant. [...]. Ma chambre était connexe avec celle d'une autre personne que je ne connaissais pas. Ce n'était pas l'environnement idéal. La première année, j'ai trouvé ça dur, pour l'appartement. Ensuite j'ai déménagé. À Montréal, ce qui a vraiment marqué mon établissement, c'est les nombreux déplacements. J'ai déménagé toutes les années. Soit parce que mon appartement ne me convenait pas, [ou que] ne me convenait pas mon coloc, ou [à cause d']une invasion de souris. Ça a été dur. (MI-34/F)

La question du logement et du quartier est illustrée par la confrontation des points de vue de ces deux jeunes en situation contrastée. Les témoignages que nous venons de voir attirent l'attention sur le fait que la qualité du logement et les caractéristiques du quartier peuvent jouer un rôle important dans la sociabilité et l'intégration des jeunes migrants à Montréal. Nous constatons également que les jeunes migrants québécois accordent de l'importance à l'environnement de leur habitat dans l'espace urbain, qui devient pour eux un espace concret où il est possible de vivre des expériences stimulantes.

Dans un premier cas, le jeune migrant exprime son enthousiasme de vivre dans un quartier animé et populaire, où toutes les formes de sociabilité sont admises, comme c'est le cas du Plateau-Mont-Royal. Dans un deuxième cas, la migrante montre que des préoccupations liées au coût du logement peuvent jouer plus ou moins fortement dans le choix de l'espace de résidence. Les jeunes plus démunis n'expriment pas d'exigences envers les quartiers. Ils habitent un espace qu'ils «choisissent» souvent en fonction d'un seul critère : la modicité du prix. Mais avec le temps, apparaît le désir de quitter ce lieu d'habitation. De telle façon que le parcours de certains jeunes est marqué par plusieurs déplacements, comme dans le cas représenté ici.

Toutefois, pour d'autres jeunes migrants qualifiés, les limites du quartier ne sont pas ressenties comme une frontière stricte; d'autres éléments vont influencer leur intégration. Pour le jeune suivant, les exigences vis-à-vis du quartier sont moindres puisque son processus d'intégration s'appuie sur la reconstruction d'un réseau de sociabilité dans le milieu d'accueil: 
Trouver un appartement a été assez simple. Le grand frère d'un de mes amis avait un superbe appartement, immense. J'aimais beaucoup cet appartement-là, ça s'est bien passé. Il y avait des gens là-dedans à qui je n'avais jamais parlé quand on était à (municipalité d'origine). On était en train de créer une version miniature de (municipalité d'origine) à Montréal. (MI-29/H)

Comme l'observent Assogba et al. (2003: 24), «le maintien des liens avec les groupes d'appartenance d'origine et la création de nouveaux réseaux sont deux phénomènes participant à l'intégration». Le jeune ci-dessus a eu d'abord une tendance à rejoindre des gens issus du même milieu d'origine que le sien à son arrivée à Montréal.

Toutefois, le sentiment d'appartenance régionale conditionne également les représentations que se font les jeunes migrants. Tout comme chez les jeunes Brésiliens, les migrants qui gardent un plus fort attachement à leur région d'origine ont tendance à être davantage affectés par les aspects négatifs de la vie en métropole.

Le fait d'appartenir à un groupe permet de développer des relations étroites avec des individus proches. La proximité spatiale des migrants provenant de la même région d'origine est un outil important dans le processus d'intégration à la métropole. Le jeune interviewé illustre qu'un sentiment de sécurité se développe dans le cas où la cohésion avec des gens du milieu d’origine se concrétise.

Les récits d'autres jeunes soulignent le fait que la dynamique propre à chaque ville peut influencer leur trajectoire d'intégration. Montréal, par exemple, a un cachet particulier. La dynamique d'une ville repose sur un ensemble d'éléments, certains matériels, d'autres symboliques, qui en font un lieu unique ne pouvant être confondu avec aucun autre. Les divers éléments qui fondent la dynamique de la ville font que les jeunes Québécois peuvent être confrontés à une multiplicité de situations, lors de leurs parcours en milieu métropolitain.

Ça a pris du temps, mais maintenant je suis adaptée à Montréal. Aujourd’hui, je vois le côté multiculturel, le bon côté de Montréal. Avant, je voyais juste le côté laid. Maintenant je peux voir le bon côté. Je me suis habituée et je me suis attachée à des coins que je connais mieux. J'ai comme accepté aussi que c'était flou, que je vais venir pour un bout de temps indéterminé. Avant, c'était «je viens, je fais mes études, je retourne». Maintenant, [c'est] «je suis bien ici». J'ai un amoureux qui est ici. J'ai un appartement mieux qu'avant. Ça coûte quand même cher, la vie c'est quand même cher aussi, mais je profite au maximum maintenant. (MI-22/F)

Je me sens un peu intégrée. Mais j'ai comme l'impression que la gestion du temps est un peu dure à Montréal, qu'il me reste moins de temps pour faire plusieurs choses. Je vais à l'école, il faut payer l'école, je travaille, j'ai un copain, j'essaie de faire un peu de sport. [Tout] ça, déjà ça a pris beaucoup de temps. Imagine-toi si j’avais des enfants, je n'aurais plus de temps pour voir mes amis. J'ai l'impression que ça passe vite. (MI-24/F)

J'ai eu plus de difficulté à m’habituer, [savoir] qui [fait quoi] à Montréal. Il y a toujours un bruit de fond, toujours la circulation, les odeurs aussi, ça, j'ai eu de la misère à m'habituer à ça. À Montréal, il y a une espèce d'odeur constante de circulation, des usines et tout ça. (MI-33/H)

Je suis content à Montréal. J'aime beaucoup ma vie aussi à Montréal. Les amis que j’ai vus passer ici, j'ai eu du fun. Il y a des injustices sociales. Je trouve ça plate, de la pauvreté. Mais elle est belle la ville aussi. (MI-40/H) 
Montréal constitue un creuset pour ces jeunes Québécois, plutôt représentatifs dans un monde de mobilité. Ainsi, cette métropole matérialise les intercommunications et rend possibles des représentations sociales et des échanges diversifiés, à l'image de toutes ses différences. Ainsi, des expressions comme «bouillonnant», «bruit», «saleté», «vie accélérée», «multiethnique», entre autres, traduisent les représentations que les jeunes se font de la ville.

Les extraits des entrevues avec ces jeunes illustrent le fait que chaque individu possède un niveau d'exigence particulier par rapport aux besoins de l'intégration dans l'espace urbain. Ces jeunes peuvent être considérés comme des individus maîtrisant bien leur espace quotidien. Mais leur insertion dans la ville ne suit pas le même chemin d'un à l'autre, car autant leur choix d'habitat que leurs activités individuelles présentent de grandes différences.

\section{Conclusion}

Les formes d'insertion des migrants dans les grandes villes sont des processus dynamiques. Nous avons tourné notre attention vers quelques dizaines de jeunes qui se sont installés à Porto Alegre ou à Montréal et nous avons examiné leur processus d’intégration dans ses dimensions économique, sociale et symbolique.

Dans la sphère économique, les jeunes Brésiliens semblent rencontrer plus de difficultés que les jeunes Québécois. Cela est dû aux faibles investissements publics dirigés vers les jeunes, au Brésil. En conséquence, les programmes qui mobilisent les jeunes autour d'activités économiques sont encore limités. Dans les pays industriels avancés, comme le Canada, les différents gouvernements élaborent des politiques sociales d'insertion socioéconomique pour les jeunes qui éprouvent des difficultés.

Sur le plan social, les difficultés d'insertion des jeunes migrants dans l'espace métropolitain sont multiples. Nous avons observé qu'elles peuvent varier selon les valeurs des jeunes, leur tissu relationnel et le sentiment d'appartenance à leur région d'origine.

En plus des difficultés éprouvées dans la sphère sociale, nous avons observé des difficultés liées à la sphère symbolique, surtout chez les jeunes Brésiliens. Les jeunes Québécois font preuve de plus d'autonomie dans leur projet migratoire. Les jeunes Brésiliens ressentent davantage de conflits entre les intérêts familiaux et les projets individuels. L'âge et le soutien parental peuvent avoir un effet sur l'autonomie migratoire des jeunes qualifiés, dans le cas brésilien. Nous avons constaté que ce degré d'autonomie peut varier selon le niveau d'attentes et d'investissements et selon la position sociale de la famille.

L’analyse des difficultés des jeunes migrants qualifiés dans ces trois sphères a révélé les différents obstacles rencontrés par les jeunes migrants brésiliens comparativement aux jeunes migrants québécois. À la lumière de cet examen, nous pouvons observer que, pour les jeunes Québécois, les principales difficultés sont issues de la sphère sociale (intégration sociale, appartenance) tandis que, pour les jeunes Brésiliens, les difficultés sont plus concentrées dans les sphères économique (contexte socioéconomique personnel) et symbolique (modèles familiaux et projets parentaux). Il 
faut également noter que les difficultés éprouvées par les jeunes dans leur trajectoire migratoire ne les affectent pas tous de la même façon. L'importance des difficultés diminue avec l'agmentation du niveau de scolarisation.

En résumé, pour les jeunes Brésiliens, nous observons que les éléments qui contribuent à leur intégration dans la métropole de Porto Alegre sont surtout liés à la solidarité familiale (soutien familial), aux liens sociaux tissés avec des amis ou des membres de la famille résidant dans la métropole, à un réseau de sociabilité avec des gens du même milieu d'origine ou encore à une expérience de départ à l'étranger déjà vécue. Parmi les éléments qui ont le plus défavorisé leur processus d’intégration, les limitations économiques, les rapports sociaux faibles avec les Porto-Alegrenses, la violence et l'individualisme sont les plus fréquemment évoqués.

Pour les Québécois, les éléments qui contribuent à l'intégration des jeunes dans la métropole de Montréal sont surtout: la bonne qualité du logement, la qualité de vie du quartier, le fait de sortir et rencontrer des gens, les réseaux de sociabilité établis avec les gens du milieu d'origine et le fait d'avoir un amoureux ou une amoureuse dans la métropole. Parmi les éléments qui freinent leur intégration, il y a les nombreux déplacements effectués entre plusieurs quartiers de la ville, le bruit dans certains espaces urbains, la difficulté de trouver un logement propre et le coût élevé de la vie dans la métropole.

La nature temporaire ou définitive du projet de migration vers la métropole est également un élément susceptible d'influencer le processus d'intégration des jeunes migrants. Pour les jeunes Brésiliens et les jeunes Québécois qui se dirigent vers Montréal ou Porto Alegre et dont le parcours migratoire est vécu comme une étape transitoire du cycle de vie et non une étape définitive, l'intégration dans la métropole comporte une certaine dose d'ambivalence et d'attentisme. Nous notons que même les jeunes intégrés (jouissant de bonnes conditions socioprofessionnelles, possédant un réseau d'amis et disposant de leur propre logement) semblent être disponibles pour une nouvelle migration. Il n'en reste pas moins que ces jeunes affichent une bonne intégration dans l'espace métropolitain, intégration qui ne se traduit pas toujours par un fort sentiment d'appartenance à la métropole.

L’appartenance régionale influence le rapport des jeunes avec le milieu métropolitain. Pour les jeunes Brésiliens, le fait d'avoir un fort sentiment d'appartenance régional ne stimule pas nécessairement leur intérêt à revenir dans leur milieu d'origine. Pour les jeunes Québécois, le fort sentiment d'appartenance joue un rôle plus positif dans le processus de la migration de retour. Toutefois, tant pour les jeunes Brésiliens que pour les jeunes Québécois, un fort sentiment d’appartenance régionale peut étirer le délai d'intégration au milieu métropolitain. Le processus d'intégration pour ces jeunes fortement attachés à leur région d'origine semble être plus difficile.

Le fait de ne pas s'attacher à la métropole peut aussi signifier de nouveaux défis à relever et s'avérer l'occasion de vivre encore de nouvelles expériences. À cet égard, la vie dans la métropole permet aux jeunes de développer une certaine autonomie, une indépendance et de la débrouillardise, qui ont aussi un effet sur leur façon d'utiliser l'espace. 
ABRAMO, Helena et BRANCO, Pedro Paulo (2005) Retratos da Juventude Brasileira: Análises de uma pesquisa nacional. São Paulo, Instituto Cidadania/Editora Fundação Perseu Abramo.

ALONSO, José et BRINCO, Ricardo (2006) Caracterização geral da Região Metropolitana de Porto Alegre (RMPA). Publié pour la FEE - Fundação de Economia e Estatistica.

ASSOGBA, Yao, FRÉCHETTE, Lucie et GAGNON, Caroline (2003) Dynamiques des trajectoires migratoires intra-régionales des jeunes en Outaouais. Une enquête qualitative. Gatineau, Université du Québec en Outaouais, Cahiers du CÉRIS.

BOUDREAULT, Pierre et PARAZELLI, Michel (dir.) (2004) L'imaginaire urbain et les jeunes: la ville comme espace d'expériences identitaires et créatrices. Québec, Presses de l'Université du Québec.

BRETON, Raymond (1994) L'appartenance progressive à une société: perspectives sur l'intégration socioculturelle des immigrants. Actes du Séminaire sur les indicateurs d'intégration des immigrants. Montréal, Centre d'études ethniques de l'Université de Montréal et Québec, ministère des Affaires internationales, de l'Immigration et des Communautés culturelles, p. 239-252.

BUJOLD, Johanne et GAUTHIER, Madeleine (1995) Les jeunes et le départ des régions: revue des travaux. Québec, Éditions de l'Institut québécois de recherche sur la culture (IQRC).

CHAMBERS, Iain (1994) Migrancy, culture, identity. Londres, Routledge.

CONSTANT, Amelie et ZIMMERMANN, Klaus (2011) Migration, ethnicity and economic integration. Dans Miroslav Jovanovic (dir.) International handbook on the economics of integration, vol. III. Cheltenham, Edward Elgar Publishing, p. 145-168.
CORREA, Silvio Marcus de Souza (2008) Brésil: une société des jeunes? Dans Nair Teles et Wanda Espirito Santo (dir.) Regard sur les jeunes du Brésil. Québec, Presses de l'Université de Laval, p. 219-223.

DESMARAIS, Danielle, ASSOGBA, Yao et FRÉCHETTE, Lucie (2001) L'intégration des jeunes adultes migrants en milieu urbain au Québec. Dans Henri Dorvil et Robert Mayer (dir.) Problèmes sociaux - Tome II Études de cas et interventions sociales. Québec, Presses de l’Université du Québec, p. 103-128.

FRÉCHETTE, Lucie, DESMARAIS, Danielle, ASSOGBA, Yao et PARÉ, Jean-Louis (2004) L'intégration des jeunes à la ville: une dynamique de repérage spatial et social. Dans Patrice LeBlanc et Marc Molgat (dir.) La migration des jeunes: aux frontières de l'espace et du temps. Québec, Éditions de l'IQRC et Presses de l'Université Laval, p. 81-105.

GARNEAU, Stéphanie (2006) Les mobilités internationales à l'ère de la globalisation. Une comparaison sociologique des carrières spatiales et des socialisations professionnelles d'étudiants français et québécois. Lyon, Université LumièreLyon 2, Faculté d'anthropologie et sociologie, thèse de doctorat non publiée.

GAUTHIER, Madeleine, MOLGAT, Marc et CÔTÉ, Serge (2001) La migration des jeunes au Québec: résultats d'un sondage auprès des 20-34 ans du Québec. Québec, Groupe de recherche sur la migration des jeunes, Centre Urbanisation, Culture et Société, INRS.

GAUTHIER, Madeleine, LEBLANC, Patrice, CÔTÉ, Serge, DESCHENAUX, Frédéric, GIRARD, Camil, LAFLAMME, Claude, MAGNAN, Marie-Odile et MOLGAT, Marc (2006) La migration des jeunes au Québec: rapport national d'un sondage 2004-2005 auprès des 20-34 ans du Québec. Québec, Groupe de recherche sur la migration des jeunes. Centre Urbanisation, Culture et Société, INRS. 
GOODMAN, Leo A. (1961) Snowball sampling. Annals of Mathematical Statistics, no 32 , p. $148-170$.

IBGE - Instituto Brasileiro de Geografia e Estatístca (2011) Carte intitulée Regiões metropolitanas 2009/2011.

INSTITUT DE LA STATISTIQUE DU QUÉBEC (2013) Profils des régions et des MRC: comparaisons interrégionales. Démographie. [En ligne]. http://stat.gouv.qc.ca

LEBLANC, Patrice (2010) Être jeune en région. Dans Miriam Fahmy (dir.) L'État du Québec 2010. Institut du Nouveau Monde. Montréal, Les éditions du Boréal, p. 427-430.

MATOS, Ralfo et BRAGA, Fernando (2005) Redes geográficas, redes sociais e movimentos da população no espaço. Dans Ralfo Matos (dir.) Espacialidades em rede: população, urbanização e migração no Brasil contemporâneo. Belo Horizonte, C/Arte, p. 111-154.

MESSIER, Camille et MAROIS, Michelle R. (1972) L'intégration urbaine des migrants de l’Est du Québec: «Les Gaspésiens de la ville». Recherches sociographiques, vol. 13, $\mathrm{n}^{\circ} 1$, p. 107-124.

MOLGAT, Marc et SAINT-LAURENT, Nathalie (2004) Attrait de la grande ville et projets d'avenir des jeunes migrants: en guise de réponse aux explications de la sociologie classique. Dans Patrice LeBlanc et Marc Molgat (dir.) La migration des jeunes: aux frontières de l'espace et du temps. Québec, Éditions de l'IQRC et Presses de l'Université Laval, p. 245-270.

OCDE (2001) Mieux vivre dans la ville: le rôle de la gouvernance métropolitaine. Paris, Organisation de coopération et de développement économiques.

OLIVEIRA,Carlos Wagner et DA MATA, Daniel (2008) Quais características das cidades determinam a atração dos migrantes qualificados. Instituto de Pesquisa Econômica Aplicada. Brasília.
PAPADEMETRIOU, Demetrios (1997) Metropolis : préparer l'avenir. Dans Marco Lombardi (dir.) Metropolis, première conférence. Milan, Fondazione Cariplo, p. $130-143$.

PEIXOTO, Nivea et MELLO, Oberon da Silva (2009) Mobilidade urbano-metropolitana na Região Metropolitana de Porto Alegre (RMPA). Publié pour la FEE - Fundação de Economia e Estatistica, p. 129-150.

PEREIRA, Viviane Mendonga (2000) O Recente processo migratório brasileiro e seus determinantes. Escola Superior de Agricultura Luiz de Queiroz. Piracicaba, Universidade de São Paulo.

PERRET, Cathy (2008) Les régions françaises face aux migrations des diplômés de l'enseignement supérieur entrant sur le marché du travail. Annales de géographie, $n^{0} 662$, p. 62-84.

POTVIN, Dominique (2006) Les jeunes adultes migrants de retour, un potentiel pour le développement de leur région d'origine. Université du Québec à Rimouski, Département sociétés, territoires et développement, thèse de doctorat non publiée.

PROFIL SOCIODÉMOGRAPHIQUE DE LA VILLE DE MONTRÉAL (2008) Québec, Montréal. [En ligne]. http://ville.montreal. qc.ca/pls/portal/docs/page/mtl_stats_fr/ media/documents/profil_sociod\%c9mo_ montrealnord.pdf

SASSEN, Saskia (1997) L'espace et l'emploi dans l'économie globale informatisée. Dans Marco Lombardi (dir.) Metropolis, première conférence. Milan, Fondazione Cariplo, p. 26-35.

WALDINGER, Roger (1997) Intégration des immigrants dans la métropole post-industrielle: un point de vue des États-Unis. Dans Marco Lombardi (dir.) Metropolis, première conférence. Milan, Fondazione Cariplo, p. 36-52. 